\title{
Linearity Measurement of 6G Receiver with One Transmission Frequency Extender Operating at $330 \mathrm{GHz}$
}

\author{
Marko E. Leinonen*, Klaus Nevala*, Nuutti Tervo*, Aarno Parssinen* \\ ${ }^{*}$ Centre for Wireless Communications, University of Oulu, Finland
}

\begin{abstract}
The future sixth-generation (6G) is envisioned to support data rates up to $1 \mathrm{Tbps}$. The operational frequencies of the $6 \mathrm{G}$ system will be expanded towards the sub-mmW and $\mathrm{THz}$ regions. The $6 \mathrm{G}$ systems will utilize directive beams, as well, to compensate increased signal attenuation between link ends. The linearity of a receiver $(\mathbf{R x})$ is one of the most significant parameters for any radio system. Traditional $\mathrm{Rx}$ linearity measurement relies on a two-tone measurement technique, which requires two dedicated RF signals and combining them to the test signal. The generation of two independent RF signals at a $300 \mathrm{GHz}$ frequency band leads to a costly and bulky solution.

This paper proposes a linearity measurement method for $6 G$ $\mathrm{Rx}$, which uses only one continuous wave transmission frequency extender. A method is proposed where the RF input signal of frequency extender is narrowband amplitude modulated (AM), generating side tones around continuous wave carrier. The carrier frequency and first side tones are used as test signals, and the linearity test is like a traditional two-tone test with unequal signals. It is shown that the carrier level can be modified by back-offing the RF input power in the frequency extender input. By varying the AM modulation index, the side tones $\square$ levels can be varied, enabling the sweep of the tone input power to perform Rx linearity measurements.

Index Terms-AM-modulation, AM-modulation index, CWsignal, power sweep, unequal signal level two-tone test, two-tone test.
\end{abstract}

\section{INTRODUCTION}

The currently operational $5 \mathrm{G}$ systems have been deployed at sub-6 GHz bands, and the network deployments to the millimeter-wave $(\mathrm{mmW})$ bands are on-going activity. The first wave fifth-generation (5G) new radio (NR) $\mathrm{mmW}$ mobile devices are available during 2020 [1]. The current highest frequency of the frequency range two (FR2) in the 5G specification Release 16 is $43.5 \mathrm{GHz}$. The $5 \mathrm{G}$ specification allows $\mathrm{mmW}$ frequencies to be extended to $52.6 \mathrm{GHz}$ in future releases [2]. Academic beyond 5G research aiming towards $6 \mathrm{G}$ systems has started, and the first networks supporting the $6 \mathrm{G}$ are expected to appear 2030 [3]. The ambitious target for the $6 \mathrm{G}$ system is to support extreme wireless data rates up to $1 \mathrm{Tbps}$. Such data rate requires a significant amount of operational spectrum, and those are available only beyond $5 \mathrm{G} \mathrm{FR} 2$ at $>100 \mathrm{GHz}$ frequencies. There are no frequency allocations available for the $6 \mathrm{G}$ systems, but one option is to utilize frequencies around $300 \mathrm{GHz}$. There is available an IEEE standard 802.15.3d, which proposes wireless fidelity (Wi-Fi) around $300 \mathrm{GHz}$ [4].

There are two main technological approaches: to use electrical integrated circuits or to use photo-electronics for the radio solutions to support envisioned $6 \mathrm{G}$ frequency allocations beyond $100 \mathrm{GHz}$ frequencies. The photo-electronic approach has large physical dimensions, and the highest reported data rate is 132 Gpbs with a link distance of $110 \mathrm{~m}$ in [5]. An $850 \mathrm{~m}$ fixed wireless link at $240 \mathrm{GHz}$ with a 64 Gbps data rate was reported in [6]. The link was based on electronic InGaAs mHEMT implementation in transmission (TX), and reception $(\mathrm{RX})$ ends. A data rate of $80 \mathrm{Gbps}$ has been reported [7] with a meter wireless link based on InP HEMT circuits.

The linearity of a receiver $(\mathrm{Rx})$ is one essential radio parameter in any wireless communication system. The traditional approach for characterizing the $\mathrm{Rx}$ linearity has been to use a two-tone test, where two equal levels of continuous wave $(\mathrm{CW}) \mathrm{RF}$ test signals, at frequencies $\mathrm{f} 1$ and $\mathrm{f} 2$, at the operational band are fed into the receiver. There are multiple options to combine two RF signals into one test signal. An RF signal generator (SG) with a six times multiplier and an additional phase-locked Gunn-oscillator is used to generate two test frequencies from 75 to $90 \mathrm{GHz}$ frequency band in [8]. A frequency extender generates a frequency $f 1$, and the RF SG with a frequency tripler is used for a frequency $f_{2}$ to generate test signals from 75 to $90 \mathrm{GHz}$ in [9]. The test signals $f_{1}$ and $f_{2}$ are combined with waveguide combiners, and the signal levels are matched with waveguide attenuators in [8], [9], which are bulky, rigid, costly, and large.

The most prominent frequencies for $6 \mathrm{G}$ are beyond current native test equipment frequencies, and thus operational frequencies of those need to be extended with frequency extenders. A test system, which supports a digitally modulated signal for conducted testing is presented in [10]. There are frequency extenders available for modulated and CW signal usage, but both have limitations. The modulated signal extenders have limitations with TX power. On the other hand, the $\mathrm{CW}$ extenders used with a vector network analyzer (VNA) have limitations on the supported signal bandwidth (BW). The block diagram of the VNA-based measurement setup, including the

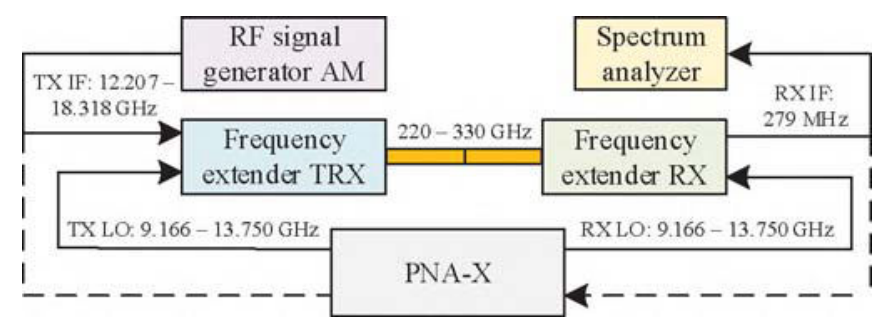

Fig. 1: Block diagram of VNA-based measurement setup using frequency extenders. 


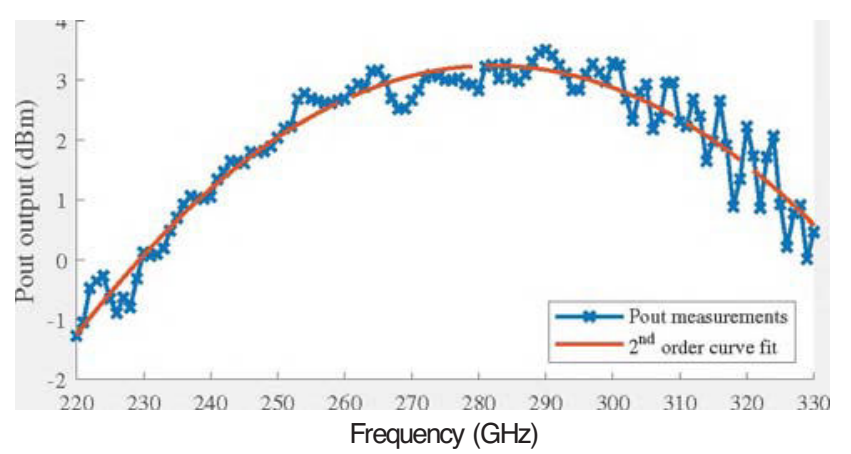

Fig. 2: Transmission power of Tx extender at $220-330 \mathrm{GHz}$ band.

$300 \mathrm{GHz}$ frequency extenders, is shown in Fig. 1. The upand down-conversion of the local oscillator (LO) frequencies can be applied and controlled from the VNA or an external SG [11]. The TX signal up-conversion to RF frequency is done by multiplying the TX intermediate frequency (IF) signal 18 times in the Tx extender. The down-conversion LO is generated by multiplying the RX LO 24 times in Rx extender, and then it is mixed with the RF signal. The TX LO is used for TX reflection coefficient measurements.

$$
\text { II. Frequency Extender Measurement setup }
$$

The RF testing at $300 \mathrm{GHz}$ frequency range requires frequency extenders, and the $\mathrm{CW}$ ones provide the highest output power. The CW frequency extenders use an RF architecture, where both RF and LO signals are multiplied several times in the RF signal chain. Thus those are very nonlinear RF devices, and the BW of the extender is limited. The Tx extender's internal signal multiplications prevent the linear combination of multiple signals due to a self-mixing. The CW Tx frequency extender has a constant TX output power when RF and LO signals are set at normal operating levels.

The CW extender's typical operation does not support the Rx's linearity measurement since the linearity measurement requires to sweep the input power levels of two different test frequencies. A proposal of usage of CW extenders for the linearity measurements is addressed in the following subsections considering the mentioned limitations.

\section{A. Output Power Control of Frequency Extender}

The transmitter (Tx) extender's output power is measured and modelled over the band of interest from $220 \mathrm{GHz}$ to $330 \mathrm{GHz}$ in Fig. 2. The TX power of the extender should be constant, but the response shows around $4.5 \mathrm{~dB}$ ripple, which is in-line with the specification in [11].

It was noticed that the TX power could be changed by adjusting the RF input power level to the Tx extender, and the measured TX control curve at $300 \mathrm{GHz}$ frequency with varied RF input power is shown in Fig. 3. However, it should be noted that the power control is highly nonlinear to the extender's input power.

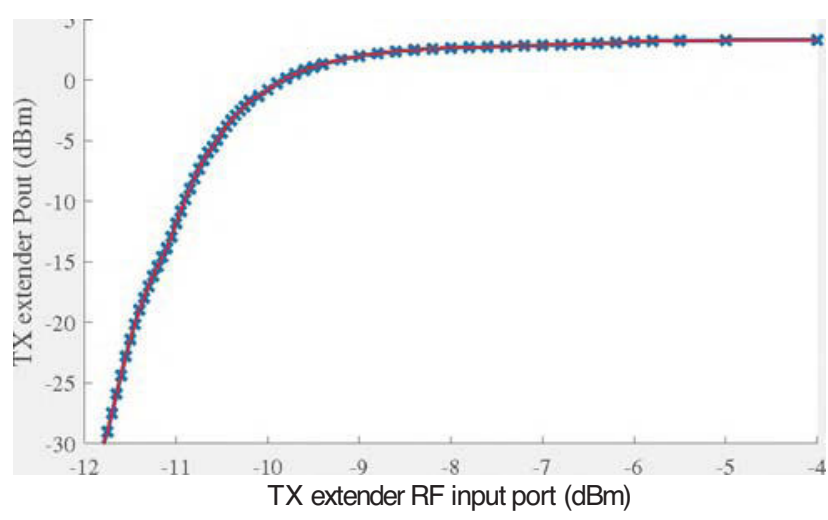

Fig. 3: Pin vs Pout of the Tx extenders at $300 \mathrm{GHz}$. The input power can be used to control the output power but the control is highly nonlinear.

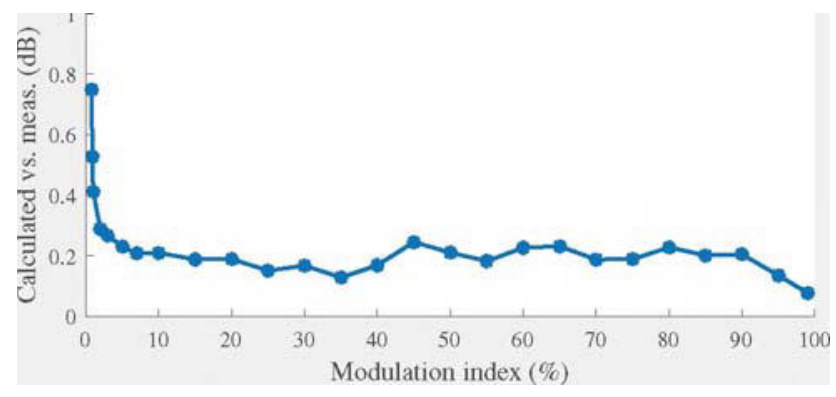

Fig. 4: AM-modulation accuracy of RF signal generator at $279 \mathrm{MHz}$.

\section{B. AM-signal Generation for Frequency Extender}

The theoretical one sided AM-modulation signal power $\mathrm{P}_{2}$ at frequency $\mathrm{f} 2$ with a known modulation index $\mathrm{m}$ is [12]

$$
\begin{gathered}
\text { ( } m \text { } \\
\text { TTOO) }
\end{gathered}
$$

where $P_{1}$ is the carrier signal power at the at frequency $f_{1}$ in $\mathrm{dBm}$ and the $\mathrm{m}$ is a percentage point. For $100 \%$ modulation $(m=1.0)$, the amplitude of each sidebands will be one-half of the carrier amplitude or $-6 \mathrm{~dB}$ below the carrier power [12].

The measured AM-modulation accuracy of the RF signal generator [13] at $279 \mathrm{MHz}$ is compared with the calculated values when the modulation index is varied from $1 \%$ to $99 \%$ is shown in Fig. 4. The measured AM-test tone level accuracy is $0.4 \mathrm{~dB}$ to the theoretical value when the modulation index is higher than $1 \%$. Thus, the measured accuracy is significantly better than the maximum $6 \%$ error in the signal generator specification [13].

\section{Third Order Intercept Point with Unequal Test Tones}

The CW frequency extenders have severe signal BW limitations due to signal multiplication RF architecture, but those can support a narrowband AM-modulation around the carrier $\mathrm{CW}$ based on measurement results. The carrier $\mathrm{CW}$ signal and the AM-modulation signal or the AM-beat can be used as test signals $\mathrm{f} 1$ and $\mathrm{f} 2$, respectively as shown in Fig. 5a. The 2 nd order non-linearity generates intermodulation (IM) to 


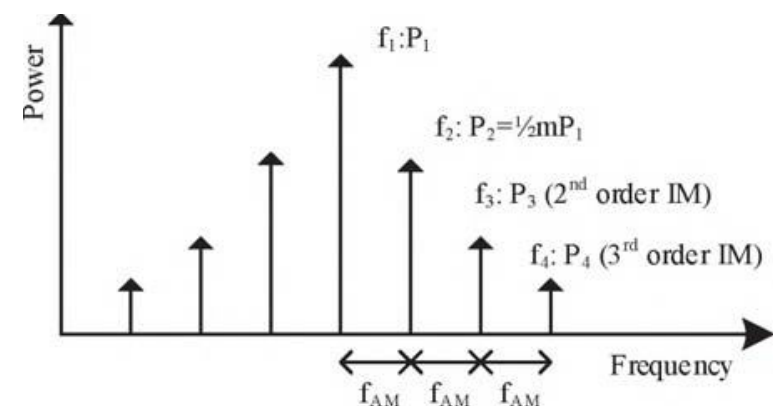

(a)

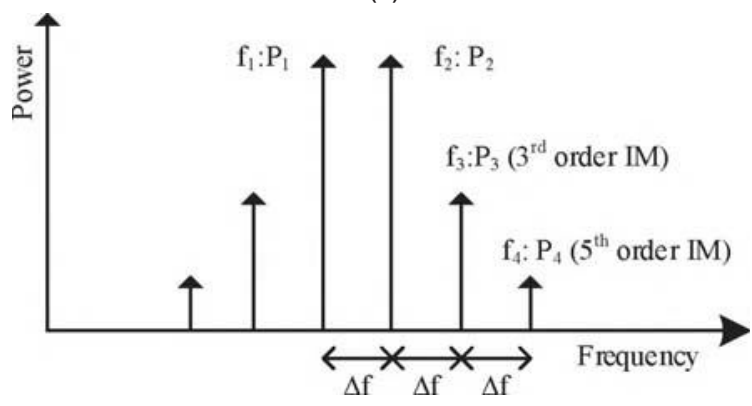

(b)

Fig. 5: Expected nonlinear components at DUT output with (a) AM modulation and (b) two tone test.

frequency $\mathrm{f} 3$ and and 3rd to $\mathrm{f} 4$. Test tones are equal powers at $\mathrm{f} 1$ and $\mathrm{f} 2$ frequencies in the traditional two-tone measurement, and the IMs at frequencies at $\mathrm{f}_{3}$ and $\mathrm{f}_{4}$ are is due to $3 \mathrm{rd}$ and 5th non-linearities as illustrated in Fig. 5b.

The input 3rd order intercept point (IIP3) for the two-tone test with unequal signals and can be calculated as in [14], [15]

$$
\begin{aligned}
& \text { Pout Pout } \\
& I I P 3=P\{n+-\wedge---(2)
\end{aligned}
$$

where $P^{\wedge}$ is input power at $\mathrm{f} 1, P 2 u$ is output power at $\mathrm{f} 2$ and $P^{\mathrm{TM}}$ 1 input power at $\mathrm{f} 3$ frequency.

The linearity of the spectrum analyzer $(\mathrm{E} 4446 \mathrm{~A})$ was measured with the traditional two-tone test. The measured IIP3 was $31 \mathrm{dBm}$, which was higher than the $28 \mathrm{dBm}$ specification [17], and the spectrum analyzer was linear enough for the receiver IIP3 measurement. The linearity of the output of the signal generator was measured with an AM-modulated signal. The TX frequency $16.666 \mathrm{GHz}$ was selected, and the RF level was set $-5.8 \mathrm{dBm}$. The AM-modulation of the RF signal was varied with the modulation index from $0.1 \%$ to $99 \%$. The AM-modulation signal level varied from $-71.8 \mathrm{dBm}$ to $-11.9 \mathrm{dBm}$ as shown in Fig. 6. The 3rd order output intercept point (OIP3) of $5.1 \mathrm{dBm}$ for the AM-modulator's linearity of RF signal generator has been measured, and measured signal levels are shown in Fig 6. similarly, the value of output 2 nd order intercept point (OIP2) is $10.1 \mathrm{dBm}$. It can be seen that the intermodulation (IM) products of 2nd and 3rd order nonlinearities are following almost perfectly the theoretical 2:1 and 3:1 slopes.

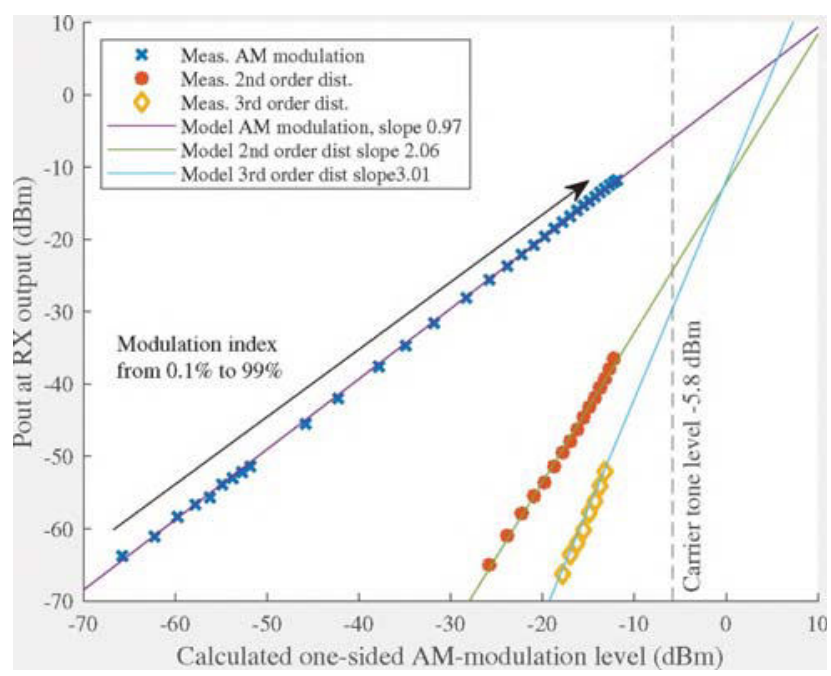

Fig. 6: Signal generator linearity with $10 \mathrm{kHz}$ AM-modulated CW signal at $16.666 \mathrm{GHz}$.

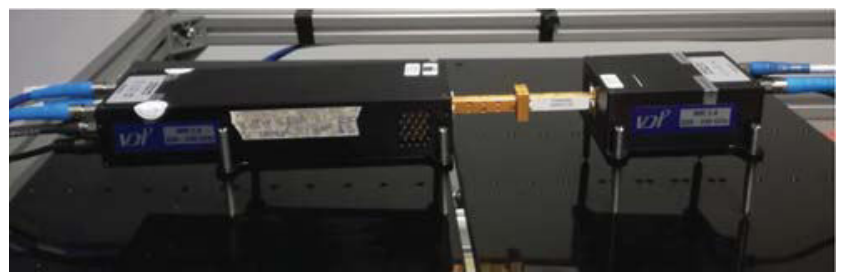

Fig. 7: Photograph of used frequency extenders for $220-330 \mathrm{GHz}$.

III. Frequency Extender Linear it y Measurements

The linearity of VNA Rx frequency extender supporting frequencies 220 - $330 \mathrm{GHz}$ [11] was measured using only one TX extender with the AM-modulation based two-tone test. The narrow band AM-modulated CW carrier passes the extender's RF signal chain linearly without significant intermodulation products. In contrast, two CW tones within the VNA frequency extender generates intermodulation distortion components over the whole spectrum the extender.

The TX IF of $16.666 \mathrm{GHz}$ was narrowband AM-modulated with a $10 \mathrm{kHz}$ modulation rate and fed into the Tx extender. The VNA controlled the LO frequencies of extenders, and the $12.51162 \mathrm{GHz}$ was used for the RX LO. The signal levels of RF and LOs were set so that the extenders operated in typical conditions. The carrier $\mathrm{CW}$ signal at Tx extender output signal was on constant level $3.3 \mathrm{dBm}$ at $300 \mathrm{GHz}$ (f1) RF frequency. The TX CW carrier level could be decreased, taking back-off from the maximum output power, as discussed in section II.

A photograph of the frequency extenders used in the measurements and those directly connected during the linearity measurement is shown in Fig. 7. The $\mathrm{Rx}$ extender module was used in a standard operation mode and, thus, a $30 \mathrm{~dB}$ waveguide attenuator in front of the Rx extender module, as shown in Fig. 7.

The Rx extender's IF output was connected to the spectrum analyzer, and the output frequency was set $279 \mathrm{MHz}$ with $\mathrm{Rx}$ 


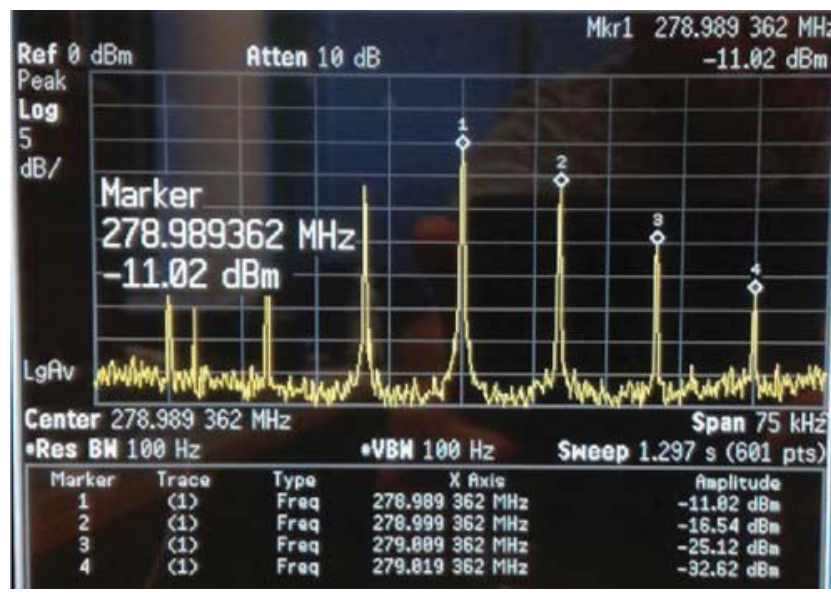

Fig. 8: Example result of AM-tones with $15 \%$ modulation index at output frequency $279 \mathrm{MHz}$ when $\mathrm{Rx}$ extender was operating at $300 \mathrm{GHz}$.

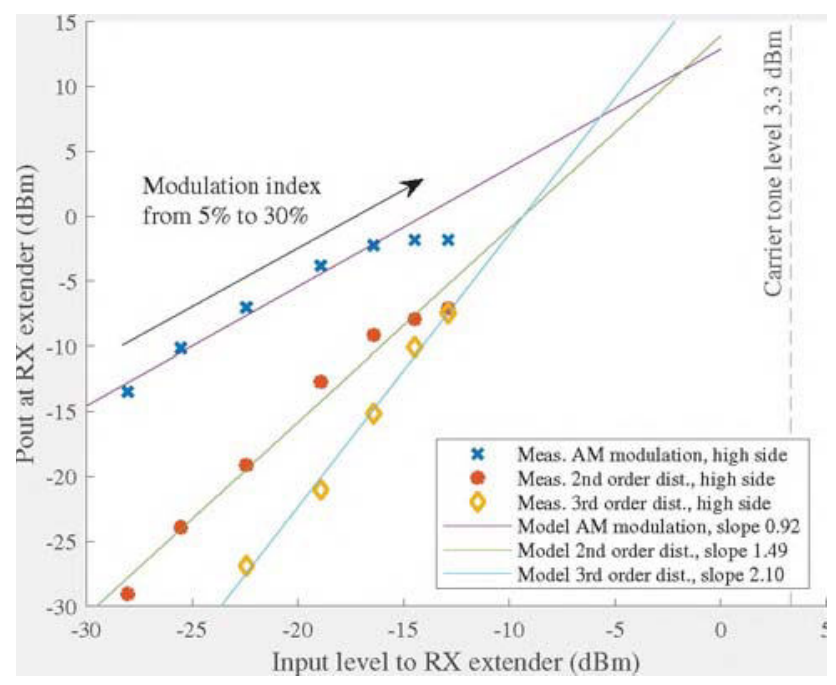

Fig. 9: Linearity measurements of directly connected waveguides of $\mathrm{Tx}$ and $\mathrm{Rx}$ extenders at $300 \mathrm{GHz}$.

LO settings. A photograph of the output spectrum from the Rx extender is shown in Fig. 8.

The linearity measurement results of the Rx frequency extender with $10 \mathrm{kHz}$ AM rate are shown in Fig. 9. The AMmodulation index was varied from $5 \%$ to $30 \%$ modulation corresponding $11 \mathrm{~dB}$ dynamic range of input signal at $\mathrm{f}_{2}$ frequency. The measured RX extender's IIP2 is $-1.8 \mathrm{dBm}$, and the IIP3 is $-5.7 \mathrm{dBm}$ based on the graphical analysis of Fig. 9 . The test signal level at $\mathrm{f} 2$ frequency saturates $-15 \mathrm{dBm}$ level, and the same phenomena can be seen for 2 nd IM results. The number of IM products and the levels start to rapidly grow from the saturation level onwards, if the modulation index is increased further.

$$
\text { IV. ConcIusion }
$$

This paper proposes an unequal level two-tone test to measure the beyond $5 G$ receivers' linearity with one VNA
TX frequency extender. The unequal two-tone signals are the $\mathrm{CW}$ carrier and the AM-modulation signal. The AM-tone level can be altered by adjusting the AM modulation index. The CW frequency Tx extender provides a constant TX power in normal operation mode. However, the TX power can be changed by back-offing the RF signal level in the Tx extender input, but accurate control is needed. The measurement system complexity is significantly reduced if only a single Tx extender is enough for the Rx linearity test.

It has been demonstrated that the AM-modulation-based linearity test works at $300 \mathrm{GHz}$ by measuring the Rx extender linearity, and the same measurement method can be applied for the $6 \mathrm{G}$ receivers when those are available.

$$
\text { Acknow ledg ment }
$$

This work has been financially supported in part by the Academy of Finland 6Genesis Flagship (grant 318927), in part Business Finland funded $5 G$ Finnish Open Research Collaboration Ecosystem (5G-FORCE), and in part Business Finland funded 5G-VIIMA. Keysight Inc. has supported the research with measurement equipment donation.

$$
\text { References }
$$

[1] Ericsson Inc., "Ericsson Mobility Report" Document number: $E A B-20: 004467$ Uen, on-line; https://www.ericsson.com/en/mobilityreport/reports, Sweden, Jun. 2020.

[2] 3GPP, "NR; Base Station (BS) radio transmission and reception (Release 16)," 3GPP, Technical Specification (TS) 38.104, 06 2020, version 16.4.0.

[3] M. Latva-aho and K. Leppanen, "Key drivers and research challenges for 6G ubiquitous wireless intelligence11 Univ. of Oulu, Finland, 2019.

[4] IEEE, "IEEE Standard for High Data Rate Wireless Multi-Media Networks-Amendment 2: $100 \mathrm{~Gb} / \mathrm{s}$ Wireless Switched Point-to-Point Physical Layer,“ IEEE Std 802.15.3d-2017, pp. 1-55, Oct 2017.

[5] T. Harter et al. "Generalized Kramers-Kronig receiver for coherent terahertz communications, 11 Nat. Photonics, pp. 601-606, Oct. 2020.

[6] I. Kallfass et al., "64 Gbit/s Transmission over $850 \mathrm{~m}$ Fixed Wireless Link at $\mathbf{2 4 0} \mathbf{~ G H z}$ Carrier Frequency," J. Infrared Milli Terahz Waves, Vol. 36, pp. 221-233, Jan. 2015.

[7] P. Rodriguez-Vazquez, M. E. Leinonen, J. Grzyb, N. Tervo, A. Parssinen and U. R. Pfeiffer, "Signal-processing Challenges in Leveraging $100 \mathrm{~Gb} / \mathrm{s}$ Wireless THz", 2nd 6G Wireless Summit Proc., pp. 1-5, Mar. 2020.

[8] Y. Hwang, "Two-Tone Intermodulation Measurement of W-band Amplifiers based on High-Linearity Frequency Down-Conversion, 11 93rd ARFTG Microw. Measurement Conf."pp. 1-4, Jun. 2019.

[9] S. Kim, R. Maurer, M. Urteaga and M. J. W. Rodwell, "A HighDynamic-Range W-Band Frequency-Conversion IC for Microwave DualConversion Receivers, 11 IEEE Compound Semicond. Integr. Circuit Symp. (CSICS) Proc., pp. 1-4, 2016

[10] Keysight Inc., "A New Sub-Terahertz Testbed for 6G Research," White paper: 7120-1082.EN, Apr. 2020.

[11] Virginia Diodes Inc., "VNA Extension Modules Operational Manual," Rev. 3, Apr. 2020.

[12] Keysight Inc., "Spectrum Analysis Amplitude and Frequency Modulation," Application Note: 5954-9130, Jul. 2014.

[13] Keysight Inc., "Agilent E8247C/57C PSG CW and Analog Signal Generators," Data sheet: 5988-7454EN, Dec. 2007.

[14] K. S. Kundert, "Introduction to RF simulation and its application," IEEE J. Solid-State Circuits, vol. 34, no. 9, pp. 1298-1319, Sept. 1999.

[15] K. S. Kundert, "Accurate and Rapid Measurement of IP2 and IP3," Citeseer, online: https://designers-guide.org/analysis/intercept-point.pdf, May, 2002. Accessed: 27.9.2020.

[16] C. J. Clark, C. P. Silva, A. A. Moulthrop and M. S. Muha, "Poweramplifier characterization using two-tone measurement technique, 11 IEEE Trans. Microw. Theory Techn., vol. 50, no. 6, pp. 1590-1602, Jun. 2002

[17] Keysight Inc., "Specifications Guide Agilent Technologies PSA Series Spectrum Analyzers, E4446A (3 Hz to 44GHz)," Data sheet, Dec. 2012. 